\title{
ANALYSIS OF DIFFERENT MODELS OF MOA SURGE ARRESTER FOR THE TRANSFORMER PROTECTION
}

\author{
Ahmed Abugalia, Mohamed Shaglouf \\ Sirte University Libya \\ *Corresponding Author email: abugalia55@yahoo.com, shaglouf@su.edu.ly
}

This is an open access article distributed under the Creative Commons Attribution License, which permits unrestricted use, distribution, and reproduction in any medium, provided the original work is properly cited

\section{ARTICLE DETAILS}

\section{Article History:}

Received 10 May 2018

Accepted 6 June 2018

Available online 10 July 2018

\section{ABSTRACT}

A summary of different mathematical models of MoA surge arrester from the previous literature, are presented in this paper. Application of the surge arrester as protective is analysis through a numerical example considering the protected substation including transformer. The static model as well as dynamic one is analyzed and compared from the point of view of protected distance.

\section{KEYWORDS}

MoA, voltage, IEEE recommended model.

\section{INTRODUCTION}

Circuit breakers, which can operate within $50 \mathrm{~ms}$, are too slow to protect against lightning or switching surges. Lightning surges can rise to peak levels within a few microseconds, and switching surges within few hundred microseconds, fast enough to destroy insulation before a circuit breaker could open. Protective devices such as surge arresters are employed, and these can be used to protect equipment insulation against transient overvoltages. These devices limit voltage to a safe level and absorb the energy from lightning and switching overvoltage.

Since years metal oxide surge arresters are well accepted for effective protection against overvoltage in air insulated substation (AIS) and (GIS). The advantage of MO- arresters are of the greatest importance for the reliability of SF6-gas insulated substations (GIS) and gas-insulated cable as well.

Metal-Oxide surge arresters generally have such characteristics that their residual voltage rises for current impulse having steeper front than standard impulse current $(8 / 20 \mu \mathrm{s})$. Rising rate of residual voltage depends on the time to crest, peak value of the current and kinds of models. The laboratory tests prove that the Metal-Oxide surge arresters have very strong dynamic V-I characteristics for lightning and other fast-front surges. These characteristics become important in considering the insulation coordination and arrester location, [1]. For example, for G.I switchgear the insulation coordination must be designed more rationally, taking into account of the protection performance of surge arresters.

\section{MATHEMATICAL MODELS OF MOA SURGE ARRESTERS}

Since 1975 when the first practical application of Metal-Oxide surge arrester to real power system took place, a number of studies and publications describes many methods of MoA modeling. Summary of some models proposed and investigated in several previous literatures is discussed in the following paragraphs.

\subsection{Nonlinear Resistors model (static model)}

The nonlinear resistor is a such device which has the property that their resistance diminishes sharply as the voltage across them increases. This

characteristic is usually expressed as: $I=K U^{\alpha}$, where $\alpha$ is the degree of nonlinearity between the current and voltage and is dependent on the MoA material [2]. The constant $K$ is likewise dependent on the material, but in addition it is controlled by geometry of the element, it is proportional to the cross-sectional area of element and inversely proportional to its length.

These kinds of models are applied in circuits at all voltages levels, for example in large chunks, under oil, across the windings of power transformer. They are connected across the apparatus to be protected and so they withstand the system voltage under nominal operation conditions. On incidence of a surge of voltage, the resistance falls rapidly as the voltage arises, thereby diverting much of the current and energy of the surge into the suppresser.

\subsection{Dynamic model}

This static characteristic can be changed to dynamic characteristic as adding a series inductance $L$. The equivalent circuit is sown in fig. (1). The value of non-linear inductance may estimate once the arrester current is known from trail run [3].

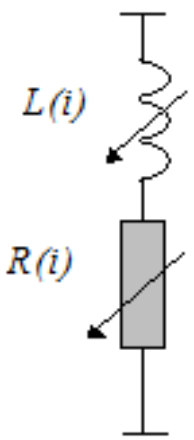

(a)

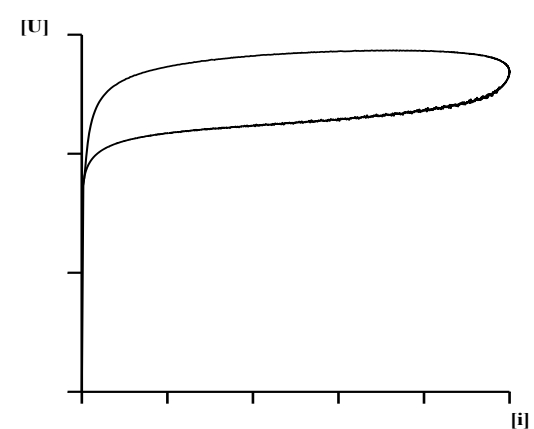

(b)
Figure 1: a) Equivalent circuit for dynamic model b) u-i characteristic

The $u-i$ characteristic of this model for a surge current has a looping tendency as shown in fig.(1-b), this loop is due to a time lag in response of MOA element, the size of loop depends on the magnitude and wave shape of current. This characteristic suggests that there is equivalently a nonlinear inductance the series with non-linear resistance. Considering static $\mathrm{v}$-i characteristic as in figure 1 , a suitable non-linear voltage current relation $U(I)$ may be expressed in dynamic characteristic. So, using this non-linear resistance, the equivalent circuit presented in figure (1-a) should characterize transient response of MOA element if the value of nonlinear inductance $\mathrm{L}$ is good chosen. 
The problem lies in that the fixed value of $L$ is not valid for all operation conditions, and this fact should be taken in the analyzing of $\mathrm{v}$-i curve $U(I)$

\subsection{IEEE Recommended Model}

The model for fast front currents surges with time to crest in the range of 0.5 to $10 \mu \mathrm{s}$ is developed by Durbak, and simulated in EMTP as shown in figure 2 [4]. The main idea is to divide a single non-linear resistance into $m$ parallel non-linear resistance, separated by low pass filters, in practical case two parallel non-linearity are sufficient. The $R_{1}-L_{1}$ circuit is the low pass filter which separates two non-linear resistance defined by $i_{0}\left(u_{0}\right)$, $i_{1}\left(u_{1}\right)$. The inductance $L_{o}$ represent the small but finite inductance associated with magnetic fields in the immediate vicinity of the surge arrester [5].

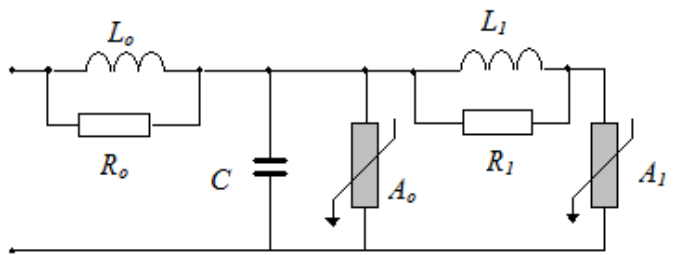

Figure 2: Recommended model of IEEE W. G.

$R_{0}$ is used only to damp numerical oscillation and $C$ is the stray capacitance of surge arrester. In the case of slow-front surges, the filter $R_{1}$ $L_{1}$ has very little impedance and the $A_{0}$ and $A_{1}$ section of the model are essentially in parallel. For fast-front surges the impedance of the $R_{1}-L_{1}$ filter becomes more significant, the result is that the arrester model active at high voltage. As general this model has many applications problems, one of them how to choose specific values of their parameters.

\section{NUMERICAL EXAMPLE}

The following example is focused on application of MoA surge arrester as a protective device. A simple model, which contains one non-linear element with static characteristic, and high dynamic model, which contains two or more non-linear elements [IEEE recommended model], are chosen. Further analysis of surge arrester efficiency as the protective device for transformer in a substation is presented.

Figure 3 represents the layout of protected substation containing transformer which is protected by surge arrester connected in parallel with it. The substation is represented by input capacity $C_{S}$ and the transformer is represented by input capacity $C_{T}$. These, approximations are allowable taking into account that the main aim of this analysis is the comparison of two models of arrester.
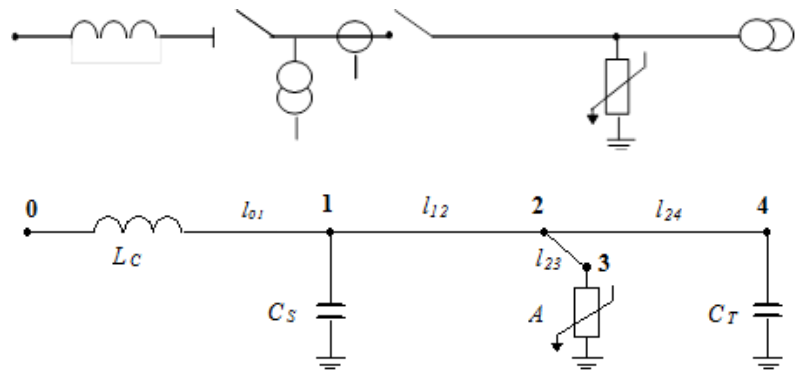

Figure 3: Layout of network of numerical example

The equivalent electric circuit of this layout is presented in figure 4.

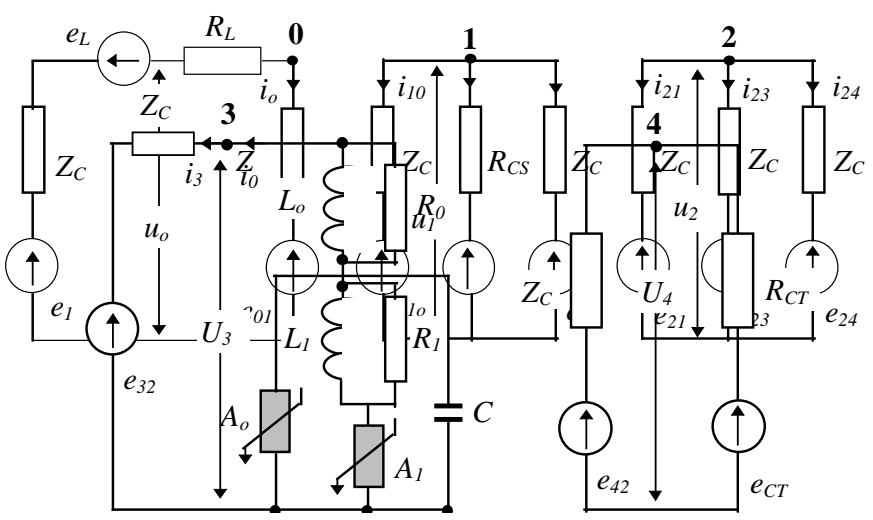

Figure 4: Equivalent circuit of network in figure 3
If we consider $t=h, 2 h, \ldots, \ldots . . ., k h$, where $\mathrm{h}$ is the time step, then, the digital solution of voltages sources of the circuit presented in fig.(4) can be written as; [6, 7]

$$
\begin{aligned}
& e_{1}=2 u_{p}(k) \\
& e_{01}(k)=u_{1}\left(k-n_{01}\right)+Z_{c} i_{10}\left(k-n_{01}\right) \\
& e_{10}(k)=u_{0}\left(k-n_{01}\right)+Z_{c} i_{0}\left(k-n_{01}\right) \\
& e_{C S}(k)=u_{1}(k-1)+R_{C S} \cdot i_{1}(k-1) \\
& e_{12}(k)=u_{2}\left(k-n_{12}\right)+Z_{C} \cdot i_{21}\left(k-n_{12}\right) \\
& e_{21}(k)=u_{1}\left(k-n_{12}\right)+Z_{C} \cdot i_{12}\left(k-n_{12}\right) \\
& e_{23}(k)=u_{3}\left(k-n_{23}\right)+Z_{C} \cdot i_{23}\left(k-n_{23}\right) \\
& e_{32}(k)=u_{2}\left(k-n_{23}\right)+Z_{C} \cdot i_{32}\left(k-n_{23}\right) \\
& e_{24}(k)=u_{4}\left(k-n_{24}\right)+Z_{C} \cdot i_{42}\left(k-n_{24}\right) \\
& e_{42}(k)=u_{4}\left(k-n_{24}\right)+Z_{C} \cdot i_{24}\left(k-n_{24}\right) \\
& e_{C T}(k)=u_{4}(k-1)-R_{C T} \cdot i_{24} \\
& e_{C}(k)=-\left[e_{1}(k)+Z_{C} i_{0}(k-1)-u_{0}(k-1)\right]-R_{L}(k-1)
\end{aligned}
$$

Where; $n_{01}=\tau_{01} / h, n_{12}=\tau_{12} / h, n_{23}=\tau_{23} / h, n_{24}=\tau_{24} / h, \tau$ is the traveling time $[\mu \mathrm{s}]$.

These equations have the following initial conditions for $k \leq 0$ :

$$
\begin{aligned}
& u_{0}(k)=u_{1}(k)=u_{3}(k)=u_{4}(k)=0 \\
& i_{01}(k)=i_{10}(k)=i_{1}(k)=i_{12}(k)=i_{21}(k)=0
\end{aligned}
$$

The digital solution of Kirchhoff's equations at connection point (0) can be obtained as follows from:

$$
\begin{aligned}
& i_{o}(k)=\left[e_{1}(k)-e_{C}(k)-e_{01}(k)\right] /\left[R_{C}+2 Z_{C}\right] \\
& \quad u_{0}(k)=e_{01}(k)+Z_{C} i_{0}(k)
\end{aligned}
$$

The digital solution of Kirchhoff's equations at connection point (1) can be obtained as follows from:

$$
\begin{aligned}
& u_{1}(k)=\left\lfloor e_{01}(k)+e_{12}(k)+e_{C S}(k) \frac{Z_{C}}{R_{C S}}\right\rfloor / \\
& \left.+\frac{Z_{C}}{R_{C S}}\right\rfloor \\
& i_{10}(k)=\frac{1}{Z_{C}}\left[u_{1}(k)-e_{10}(k)\right] \\
& i_{1}(k)=\frac{1}{R_{C S}}\left[u_{1}(k)-e_{C S}(k)\right] \\
& i_{12}(k)=\frac{1}{Z_{C}}\left[u_{1}(k)-e_{12}(k)\right]
\end{aligned}
$$$$
2+\frac{Z_{C}}{R_{C S}} \mid
$$

The digital solution of Kirchhoff's equations at connection point (2) can be obtained as follows from: 


$$
\begin{aligned}
& u_{2}(k)=\frac{1}{3}\left[e_{12}(k)+e_{23}(k)+e_{24}(k)\right] \\
& i_{12}(k)=\frac{1}{Z_{C}}\left[u_{2}(k)-e_{21}(k)\right] \\
& i_{23}(k)=\frac{1}{Z_{C}}\left[u_{2}(k)-e_{23}(k)\right] \\
& i_{24}(k)=\frac{1}{Z_{C}}\left[u_{2}(k)-e_{24}(k)\right]
\end{aligned}
$$

The digital solution of Kirchhoff's equations at connection point (3) at which surge arrester is stated are depended on its model. For static model the equations at point (3) can be written as:

$$
\begin{aligned}
& u_{a}(k)-e_{32}(k)-Z_{C} i_{a}(k)=0 \\
& u_{a}(k)=A \cdot i_{a}^{\alpha}(k) \\
& u_{3}(k)=u_{a}(k) \\
& i_{3}(k)=-i_{a}(k)
\end{aligned}
$$

For the IEEE model the equations at connection point (3) can be written as;

$$
\begin{aligned}
& \frac{e_{32}(k)-e_{32}(k-1)}{h}-Z_{C}\left\lfloor\frac{i_{L 0}(k)-i_{L_{0}}(k-1)}{h}+\frac{i_{R_{0}}(k)-i_{R_{0}}(k-1)}{h}\right\rfloor \\
& -R_{0} \frac{i_{0}(k)-i_{0}(k-1)}{h}-\frac{i_{C}(k)+i_{C}(k-1)}{2 * C}=0
\end{aligned}
$$$$
\frac{i_{C}(k)+i_{C}(k-1)}{2 * C}-R_{A_{0}}(k) \frac{i_{A_{0}}(k)-i_{A_{0}}(k-1)}{h}=0
$$

$R_{A_{0}}(k) \frac{i_{A_{0}}(k)-i_{A_{0}}(k-l)}{2}-R_{A_{l}}(k) \frac{i_{A_{l}}(k)-i_{A_{l}}(k-l)}{2}$

$-R_{l}(k) \frac{i_{l}(k)+i_{l}(k-1)}{2}=0$

$$
\begin{aligned}
& \left.L_{0} \mid \frac{i_{R_{0}}(k)-i_{R_{0}}(k-1)}{h}-\frac{i_{C}(k)-i_{C}(k-1)}{h}-\frac{i_{A_{0}}(k)-i_{A_{0}}(k-1)}{h}-\frac{i_{L_{t}}(k)-i_{L_{L}}(k-1)}{h}\right] \\
& +R_{0} \frac{i_{R_{0}}(k)+i_{R_{0}}(k-1)}{2}=0 \\
& L_{l}\left\lfloor\frac{i_{R_{I}}(k)-i_{R_{l}}(k-l)}{h}-\frac{i_{A_{I}}(k)-i_{A}(k-l)}{h}\right\rfloor+R_{I} \frac{i_{R_{I}}(k)+i_{R_{I}}(k-l)}{2}=0 \\
& R_{A_{0}}=\frac{A_{00} \cdot i_{A_{0}}^{\alpha_{o}}}{i_{A_{0}}} \quad ; R_{A_{l}}=\frac{A_{11} \cdot i_{A_{l}}^{\alpha_{l}}}{i_{A_{l}}}
\end{aligned}
$$

The digital solution of Kirchhoff's equations at connection point (4), at which the transformer is installed, can be obtained as follows from:

$$
\begin{aligned}
& i_{42}(k)=\left[e_{C T}(k)-e_{42}(k)\right] /\left[R_{C T}+Z_{C}\right] \\
& u_{4}(k)=u_{t}(k)=e_{42}(k)+Z_{C} i_{42}(k)
\end{aligned}
$$

\section{DATA APPROXIMATION AND RESULTS}

The surge voltage impulse wave $1.2 / 50 \mu$ s is approached to the substation node from infinite loss-less line, the surge is modeled according to standard wave form as [8]:

$$
u(t)=1.0534 U_{m}\left\{\exp \left(-1.478 \times 10^{4} t\right)-\exp \left(-1.653 \times 10^{6} t\right)\right\}
$$

A $100-\mathrm{kV}$ transmission line, with $550 \mathrm{kV}$ maximal testing voltage [BIL] is considering. Substation parameters are taken as $C_{S}=0.8 \mathrm{nF}$ and $C_{T}=1.2 \mathrm{nF}$. Metal-Oxide surge arrester type ABB Exlim T AM 123 is used with parameters which calculated from manufacture catalogue as: $\alpha=0.05950$ and $K=114.463 \mathrm{kV}$ for static model [9]. The IEEE model parameters are evaluated from the recommended equations $[5,10]$. The electrical circuit parameters are taken as $L_{C}=0.05 \mathrm{mH}, l_{01}=l_{12}=9 \mathrm{~m}$, and the distance Between the surge arrester and transformer is taken as $9 \mathrm{~m}$ and $18 \mathrm{~m}$. The output voltages and currents are observed at the transformer and surge arrester. The computer program is prepared to solve the problem and transient simulation results are plotted in figure 5 . a)

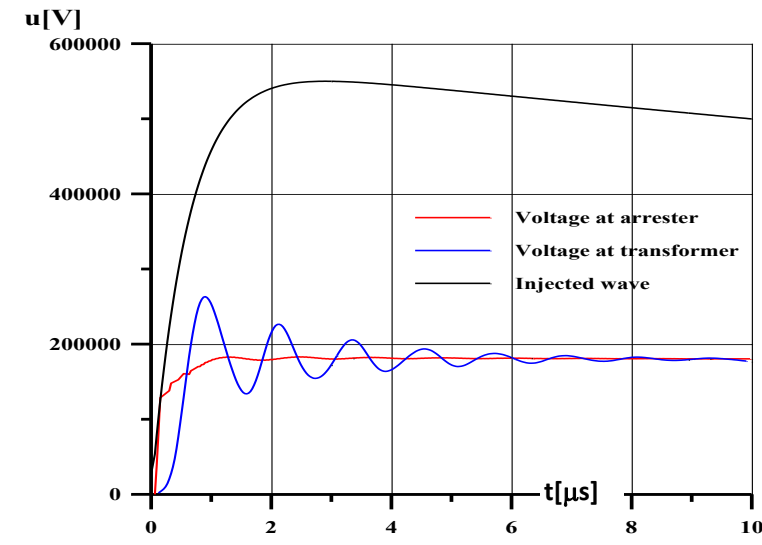

(Static model)
$\mathbf{u}[\mathbf{V}]$

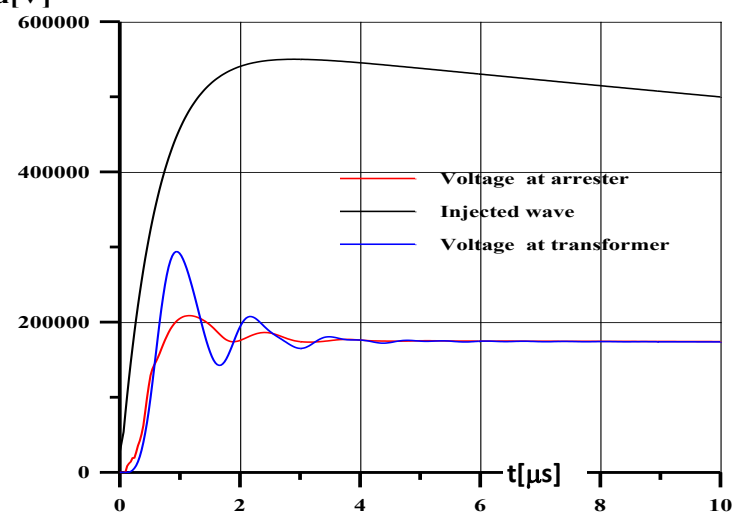

(IEEE model) 
b)

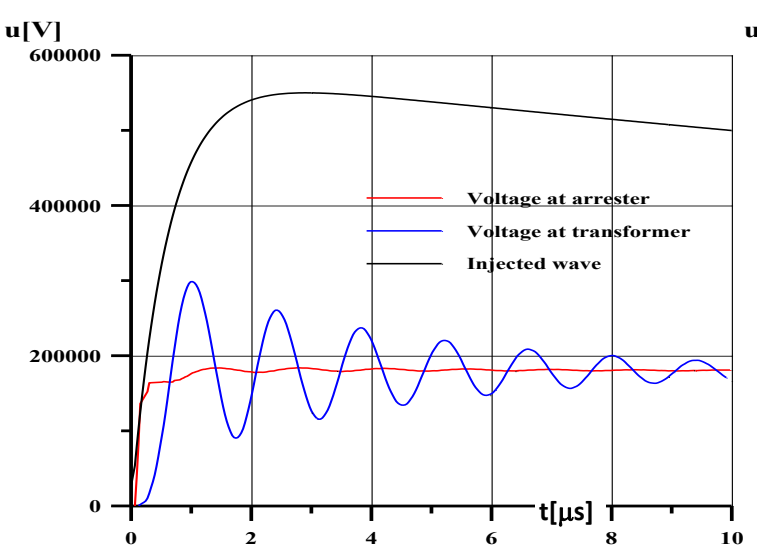

(Static model)

$\mathbf{u}[\mathbf{V}]$

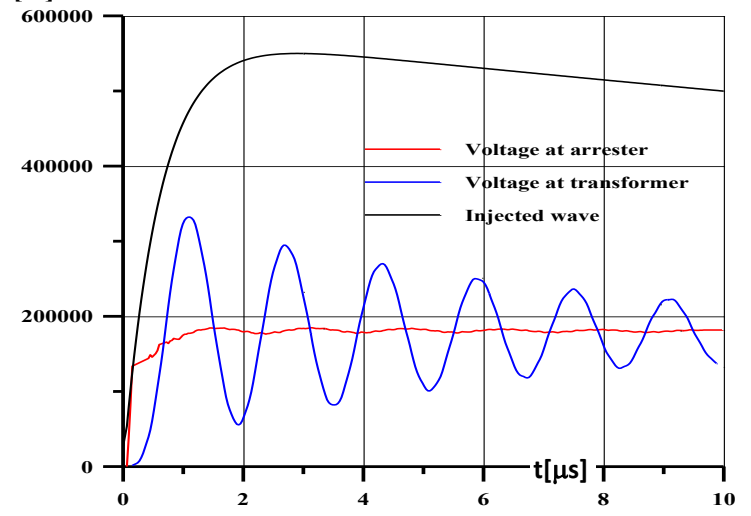

(Static model)
$\mathbf{u}[\mathbf{V}]$

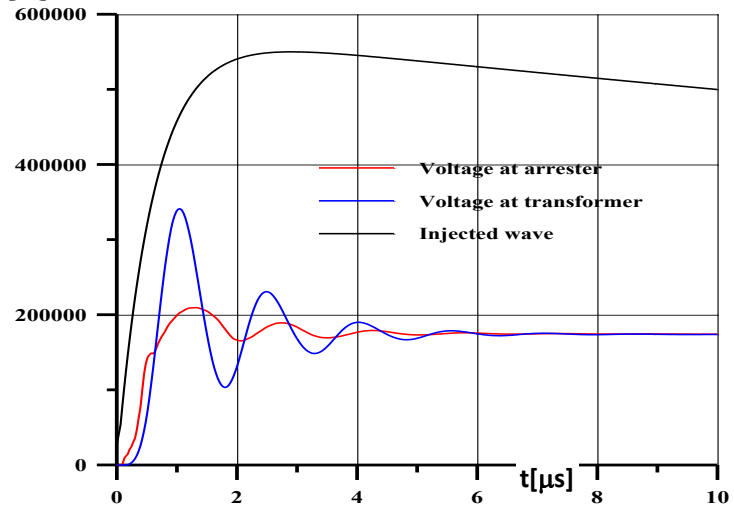

(IEEE model)

$\mathbf{u}[\mathbf{V}]$

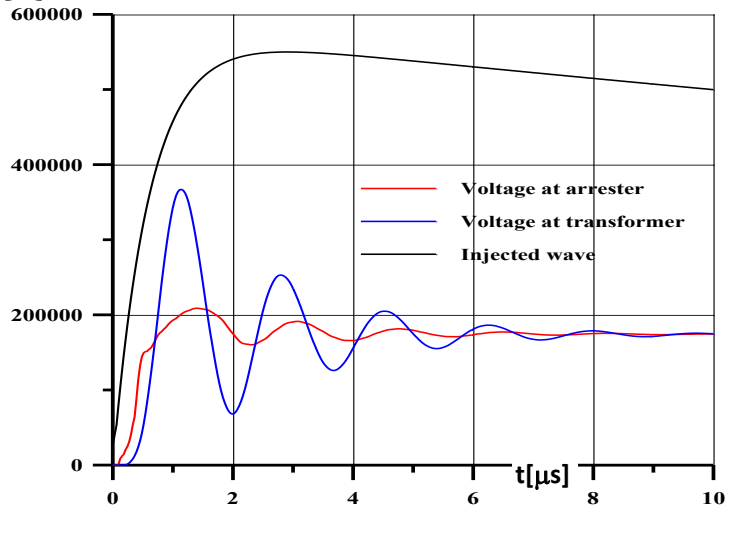

(IEEE model)

Figure 5: Comaring between the models results for surge arrester type Exlim T AM 123 at a) 9 m, b) 18 m, c) $27 \mathrm{~m}$ from connection point.

\section{DISCUSSION AND CONCLUSION}

Special two models are presented in this paper to illustrate the influence of surge arresters and their applications as protective devices for equipment like transformer through the transient computer program. From the results obtained the following points can be expressed.

1. The action of surge arrester for the overvoltage surge observed at transformer should prevent the surge magnitude exceeding the protective level, and this is verifying according the computed results.

2. For a certain time [few microseconds], the oscillations have been observed on the voltage at transformer, that is due to the wave propagation along the line and the value of wave impedance. The peak value of voltage at transformer should be not exceed the value of basic insulation level voltage [BIL], which verify in the computation results.

3. The peak of overvoltage wave observed at transformer is increase by increasing the distance between the transformer and surge arrester. This gives a good match point with the fact which recommends that the installation of surge arrester should be as nearest as possible to the protected equipment.

4. The dynamic model gives better characteristic level of protection than the static one.

\section{REFERENCES}

[1] Bajorek, J., Knott, M., Wysderka, S. 1992. Efficiency of ZnO Arrester Models in Digital Simulation of Lightning Over-voltages. Proceeding of 18th International conference on lightning Protection, Berlin Sep. 21-25, 265-270.

[2] Al-Tai, M.A., Maniatt, H.R. 1994. Protection of Transmission System against single and multiple stroke Lightning Overvoltage. 22nd International Conference on lightning Protection, 6b-06, Budapest.

[3] Tominaga, S., Azumi, K., Shibuya, Y., Imataki, M., Fujiwara, Y., Nishida,
S. 1979. Protective Performance of Metal Oxide Surge Arrester Based on the Dynamic V-I Characteristics. IEEE Transaction on Power Apparatus and System, 98 (6), 1860-1868.

[4] Durbak, D.W. 1985. Zinc-Oxide Arrester Model for Fast Surges. EMTP Newsletter, 5 (1), 1-9.

[5] IEEE working Group. 1992. Modeling of Metal Oxide Surge Arresters. IEEE Trans. On Power Delivery, 7 (1), 302-309.

[6] Wlodek, R., Bajorek, J. 1990. Examples of Overvoltage Calculations in Power Systems. Ed. AGH Krakow, (in Polish).

[7] Wlodek, R., Abugalia, A. 1998. Analysis of the Influence of Arrester Parameters on Characteristics of Transformer Protection. Scientific Quarterly of Academy of Mining and Metallurgy, Electrotechnika, Cracow (in printing) IEC Publication 801-1,2,3 1984.

[9] Switchgear, ABB HV. 1991. Catalogue, Publ. SESWG/A 2240 E, Edition 1-02.

[10] Furgal J. 1996. Computer program based on TURBO PASCAL is used to evaluate the value of voltage at surge arrester and transformer. Transient simulation results are plotted in next figures. Application of Selected Substitute Scheme for Modelling of Mo-Surge Arresters with Different Parameters. Polish Symposium: High Voltage Eng. Poznan, IW96, 19-22, 317-322. 